\title{
Prevalence and 25 year incidence of proliferative retinopathy among Danish type 1 diabetic patients
}

\author{
J. Grauslund • A. Green • A. K. Sjølie
}

Received: 8 April 2009 / Accepted: 16 June 2009 / Published online: 12 July 2009

(C) Springer-Verlag 2009

\begin{abstract}
Aims/hypothesis This study aimed to evaluate the prevalence of retinopathy in long-surviving type 1 diabetic patients. It also investigated the 25 year incidence of proliferative retinopathy and associated risk factors in a Danish population-based cohort.

Methods A population-based cohort of 727 type 1 diabetic patients from Fyn County, Denmark, was identified in 1973. In 1981-1982, baseline retinopathy was graded and other risk factors were assessed in 573 patients. Twentyfive years later, 308 patients were still alive. Of these, 201 $(65.3 \%)$ were re-examined at follow-up in 2007-2008.

Results The median age and duration of diabetes at followup were 58.8 and 43 years, respectively. At follow-up, the prevalence of diabetic retinopathy was $97.0 \%$. Nonproliferative retinopathy was found in $45.8 \%$, and $51.2 \%$ had proliferative retinopathy. The 25 year incidence of proliferative retinopathy was $42.9 \%$ among patients at risk. In a multivariate analysis, baseline $\mathrm{HbA}_{1}$ (OR 2.14 per $1 \%$ increase, 95\% CI 1.06-4.31) and non-proliferative retinopathy (OR 4.61, 95\% CI 1.94-11.0) were the only risk factors for incident proliferative retinopathy. The long-term
\end{abstract}

J. Grauslund $(\bowtie)$

Department of Ophthalmology, Odense University Hospital,

Sdr. Boulevard 29,

DK-5000 Odense C, Denmark

e-mail: jakob.grauslund@ouh.regionsyddanmark.dk

A. Green

Center for National Clinical Databases, South,

Odense University Hospital,

Odense, Denmark

\section{A. K. Sjølie}

Department of Ophthalmology, Odense University Hospital, Odense, Denmark incidence of proliferative retinopathy was not associated with baseline duration of diabetes, proteinuria, smoking, body mass index, maculopathy or systolic or diastolic blood pressure.

Conclusions/interpretation Retinopathy among longsurviving type 1 diabetic patients is almost universal. Proliferative retinopathy was found in half of these patients. In addition, the 25 year incidence of proliferative retinopathy was high. Baseline glycaemic regulation and nonproliferative retinopathy were identified as risk factors for incident proliferative retinopathy.

Keywords Diabetic retinopathy - Glycaemic regulation · Non-proliferative diabetic retinopathy · Proliferative diabetic retinopathy $\cdot$ Risk factors - Type 1 diabetes

\section{Abbreviations \\ ETDRS Early Treatment Diabetic Retinopathy Study \\ WESDR Wisconsin Epidemiologic Study of Diabetic Retinopathy}

\section{Introduction}

Diabetic retinopathy is the most common complication of type 1 diabetes [1-3] as well as the leading cause of blindness in the working-age population of the western world [3, 4]. As part of the Wisconsin Epidemiologic Study of Diabetic Retinopathy (WESDR), the 25 year progression of diabetic retinopathy was evaluated in a recent paper by Klein and co-workers [5]. The progression of diabetic retinopathy and the related risk factors were evaluated in a population-based cohort of 955 type 1 diabetic patients. Progression of diabetic retinopathy and progression to 
proliferative diabetic retinopathy were found in $83 \%$ and $42 \%$, respectively. Risk factors for incident proliferative retinopathy were poor glycaemic regulation, high systolic blood pressure, proteinuria and higher body mass index. For instance, the 25 year risk of proliferative retinopathy increased by $38 \%$ for each $1 \%$ increase in baseline $\mathrm{HbA}_{1}$.

European results comparable with those obtained from the American WESDR are lacking. Consequently, the aim of this study was to estimate the prevalence of diabetic retinopathy as well as the 25 year incidence of and associated risk factors for proliferative retinopathy in a population-based cohort of Danish type 1 diabetic patients.

\section{Methods}

Cohort and baseline examination As previously described $[6,7]$, all type 1 diabetic patients from Fyn County, Denmark, with diabetes onset before the age of 30 years $(n=727)$ were identified based on insulin prescription as of 1 July 1973. In 1973 Fyn County had approximately 450,000 inhabitants and was considered a representative 9\% sample of the Danish population [7]. In 1981-1982, 577 of 627 surviving patients $(92.0 \%)$ participated in a clinical baseline examination. Data were later lost on four patients, leaving baseline data available for 573 patients (Fig. 1). All patients gave a written informed consent at baseline as well as at the follow-up examination.

At the baseline examination, patients underwent a structured interview and ophthalmological and clinical examinations were performed [6]. BMI was calculated, and blood pressure was measured using an Erkameter Sphygmomanometer (Morton Medical, London, UK) on one arm with the patient in sitting position after $10 \mathrm{~min}$ of rest. Blood measurements included $\mathrm{HbA}_{1 \mathrm{c}}$ as total $\mathrm{HbA}_{1}$ (normal upper limit $8.1 \%$ ) with resin 70 (Bio-Rad, Hercules, CA, USA) at $20^{\circ} \mathrm{C}$ and $\mathrm{pH}$ 6.7. Proteinuria was considered present if more than or equal to $0.5 \mathrm{~g} / \mathrm{l}$ protein was found in a spot urine sample taken at any time during the day. The smoking habits of the patients were noted. Current and ex-smokers were considered to be smokers for the models.

Using tropicamide 1\% (vol.vol.), both pupils were dilated and a slit-lamp examination performed (Haag-Streit, Wedel, Germany). Ophthalmoscopy was performed and retinopathy was described and classified by a single trained observer. The patient's level of retinopathy was determined by the worst eye and classed as non-proliferative diabetic retinopathy or proliferative retinopathy, if present. Nonproliferative retinopathy included microaneurysms, haemorrhages, hard exudates, cotton-wool spots, venous beading or intraretinal microvascular abnormalities in the retina. Proliferative diabetic retinopathy was defined as

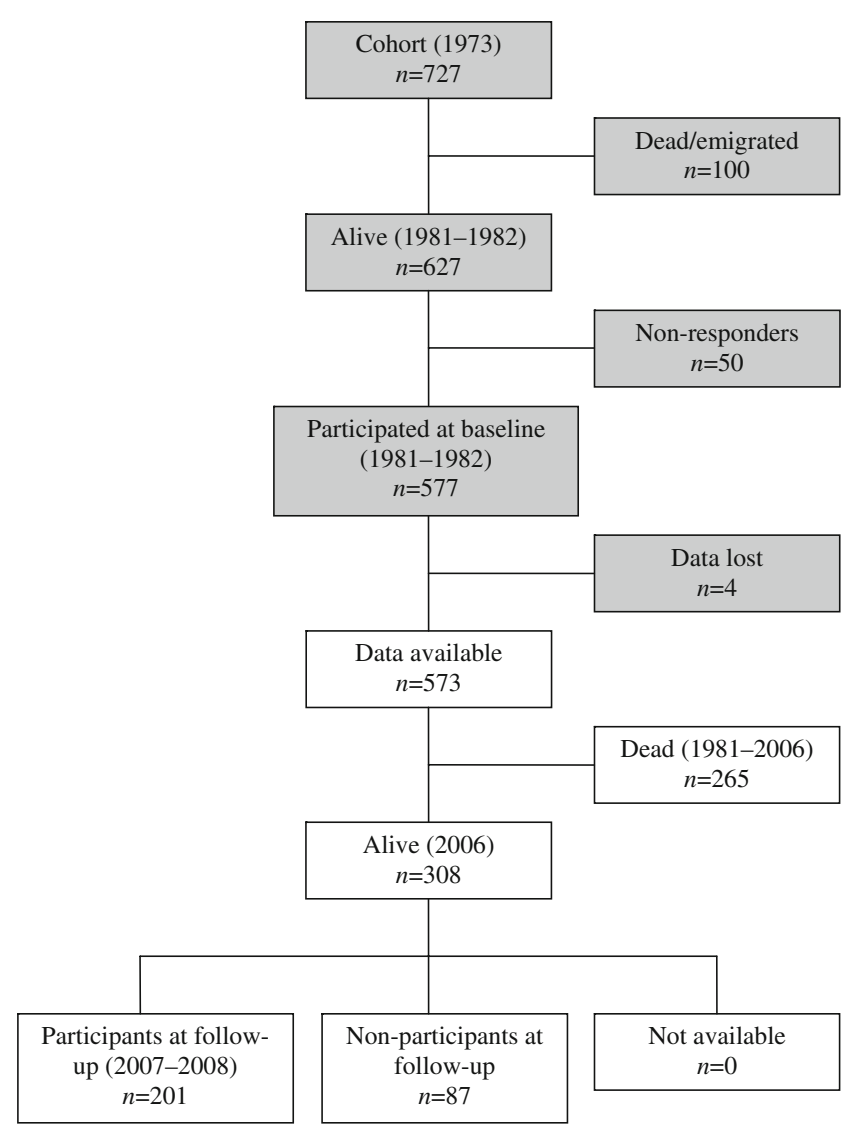

Fig. 1 Numbers of patients for whom data were available at different stages of the study

newly formed vessels in addition to the above. Furthermore, the presence of photocoagulation scars from panretinal treatment was graded as proliferative retinopathy. Maculopathy was defined as a retinal thickening in the macular area and/or the presence of perifoveal hard exudates.

Follow-up examination The follow-up examinations took place between 1 March 2007 and 1 March 2008. A full medical history was obtained. Blood pressure was measured on one arm in sitting position after 10 min of rest. An Omron M4 (Omron, Matsusaka, Japan) was used and the mean value of the last two of three measurements was used. A history of smoking was obtained. Measurement of $\mathrm{HbA}_{1 \mathrm{c}}$ was performed by cation exchange chromatography using Tosoh G8 HPLC equipment (Medinor, Broendby, Denmark) with reagents as recommended by the supplier. Normal upper limit of $\mathrm{HbA}_{1 \mathrm{c}}$ was $6.3 \%$. Nephropathy was evaluated in a single spot urine sample taken at any time during the day. Definitions were: normoalbuminuria 0-19 mg/l, microalbuminuria 20-200 mg/l and macroalbuminuria $201 \mathrm{mg} / \mathrm{l}$ and above. A monofilament test (Bailey Instruments, Manchester, UK) was used to test for neuropathy. Data on macrovascular disease were obtained 
from the Danish National Patient Registry and defined as follows: a history of stroke, myocardial infarction, angina pectoris, percutaneous coronary intervention, coronary bypass surgery or limb amputation.

Pupils were dilated and fundus photographs were taken in both eyes. Nine $45^{\circ}$ colour fields were captured with Topcon TRC-NW6S (Topcon, Tokyo, Japan) and automosaicked with IMAGEnet. Photos were graded using grading protocols $[5,8,9]$ according to the Early Treatment Diabetic Retinopathy Study (ETDRS) adaptation of the modified Airlie House classification of diabetic retinopathy $[10,11]$.

ETDRS levels were as follows: 10, no retinopathy; 20-37, mild retinopathy (microaneurysms alone or with hard exudates, cotton-wool spots and/or retinal haemorrhages); 43-47, moderate non-proliferative retinopathy (microaneurysms and intraretinal microvascular abnormalities, moderate/severe retinal haemorrhages or venous beading); 60-71, proliferative retinopathy or scars of photocoagulation; 85, end-stage proliferative retinopathy (macula obscured by haemorrhage, retinal detachment at centre of macula, phthisis bulbi or enucleation secondary to complications of diabetic retinopathy). The final category was determined by the score for the worst eye.

All images were graded at the Ocular Epidemiology Reading Center, Madison, WI, USA. Inter- and intraobserver variations and the validity of the systems have been presented elsewhere [8-10, 12].

Statistical analyses Continuous data are presented as means with standard deviations, and categorical data are presented as percentages. All statistical differences were tested at the 0.05 level. Confidence intervals that did not cross 1.0 were considered statistically significant. For categorical data, $\chi^{2}$ tests were used; for continuous data the Mann-Whitney and Kruskal-Wallis tests were used to test for differences between two and several groups, respectively.

Logistic regression analyses were performed to calculate ORs for incident proliferative retinopathy between baseline and follow-up. All patients without proliferative retinopathy at baseline who participated at follow-up were considered at risk. ORs were evaluated in an age- and sex-adjusted model as well as a multivariate model. Stata Intercooled 9.2 was used for all analyses.

\section{Results}

Of the 573 patients who participated at baseline in 19811982, 265 (46.2\%) had died before November 2006. Of the remaining 308 patients, 20 (6.5\%) could not be invited to the follow-up examination because they had emigrated or their address was unknown. Of the remaining patients, 201
(65.3\%) participated in the follow-up examination and 87 patients $(28.2 \%)$ refused to take part (Fig. 1). The participants' characteristics at the baseline examination are presented in Table 1. Patients who died before the followup examination were older, had a longer duration of diabetes, and were more likely to have proteinuria, a history of smoking, a higher systolic blood pressure, a higher level of retinopathy and a higher rate of maculopathy than patients who were still alive. The proportion of women was higher among those who chose not to participate than among the deceased and the follow-up participants.

Mean age and duration of diabetes at follow-up were 58.9 and 44 years, respectively (Table 2). Some sex-specific differences were found. A higher rate of men had a history of smoking and the diastolic blood pressure was also higher in men. As in the general population [13], women had a higher level of total cholesterol and HDL-cholesterol. At follow-up, the level of retinopathy was graded in all patients (Table 3 ). No retinopathy was found in six patients $(3.0 \%)$, non-proliferative retinopathy occurred in 92 patients $(45.8 \%)$, and 103 patients $(52.9 \%)$ had proliferative retinopathy. There were no differences between men and women.

In follow-up participants $(n=201), 33$ already had proliferative retinopathy at baseline. Consequently 168 were at risk of developing proliferative retinopathy during follow-up. Of these, 60 had no retinopathy and 108 had non-proliferative retinopathy at baseline. The 25 year incidence of proliferative retinopathy was $42.9 \%$ for all patients at risk and was significantly higher among patients who had already non-proliferative retinopathy at baseline compared with patients without retinopathy $(52.8 \%$ vs $25.0 \%, p<0.001)$.

A logistic regression was performed to identify risk factors for incident proliferative retinopathy (Table 4). In a multiple regression, baseline $\mathrm{HbA}_{1}$ (OR 2.14 per $1 \%$ increase, 95\% CI 1.06-4.31) and non-proliferative retinopathy (OR 4.61, 95\% CI 1.94-11.0) were the only risk factors identified. Duration of diabetes, proteinuria, smoking, systolic and diastolic blood pressure, BMI and maculopathy were not statistically significantly associated with development of proliferative retinopathy in the ageand sex-adjusted or the multivariate model.

\section{Discussion}

A large population-based cohort of Danish type 1 diabetic patients was followed for 25 years. Diabetic retinopathy was prevalent in long-term survivors, occurring in almost all patients; the cumulative incidence of proliferative retinopathy was $42.9 \%$. Hyperglycaemia and nonproliferative diabetic retinopathy at baseline were identified 
Table 1 Baseline characteristics (1981-1982) of patients according to status at follow-up (2007-2008)

\begin{tabular}{|c|c|c|c|c|}
\hline Characteristic & Dead $(n=265)$ & Non-participants $(n=87)$ & Participants $(n=201)$ & $p$ value \\
\hline Sex (\% male $)$ & 58.5 & 43.7 & 60.2 & 0.03 \\
\hline Age (years) & $45.9 \pm 12.6$ & $32.1 \pm 10.5$ & $33.0 \pm 10.1$ & $<0.001$ \\
\hline Duration of diabetes (years) & $28 \pm 12$ & $18 \pm 8$ & $18 \pm 8$ & $<0.001$ \\
\hline $\mathrm{HbA}_{1}(\%)$ & $8.5 \pm 1.1$ & $8.7 \pm 0.7$ & $8.6 \pm 0.7$ & 0.60 \\
\hline Proteinuria (\%) & 21.8 & 5.0 & 4.6 & $<0.001$ \\
\hline History of smoking (\%) & 78.5 & 51.7 & 57.2 & $<0.001$ \\
\hline Systolic BP (mmHg) & $155 \pm 25$ & $141 \pm 22$ & $142 \pm 21$ & $<0.001$ \\
\hline Diastolic BP (mmHg) & $95 \pm 14$ & $93 \pm 14$ & $92 \pm 12$ & 0.26 \\
\hline $\operatorname{BMI}\left(\mathrm{kg} / \mathrm{m}^{2}\right)$ & $22.7 \pm 3.1$ & $22.3 \pm 2.4$ & $22.3 \pm 2.5$ & 0.63 \\
\hline Level of retinopathy (\%) & & & & $<0.001$ \\
\hline None & 18.1 & 33.3 & 29.9 & \\
\hline Non-proliferative & 46.0 & 54.0 & 53.7 & \\
\hline Proliferative & 35.9 & 12.6 & 16.4 & \\
\hline Maculopathy (\%) & 29.1 & 9.2 & 12.9 & $<0.001$ \\
\hline
\end{tabular}

Continuous data are presented as means with standard deviations; categorical data are presented as percentages

as predictors of incident proliferative retinopathy whereas other markers such as blood pressure, proteinuria, smoking and maculopathy were not.

The 25 year cumulative incidence of proliferative retinopathy was similar to the $42 \%$ figure found in the WESDR population [5]. A high long-term incidence of proliferative retinopathy has been confirmed in other studies [14-16]. However, in a Norwegian cohort Skrivarhaug et al. reports a 25 year cumulative incidence of proliferative retinopathy of only $10.9 \%$ [17]. Given the ethnic similarities between the Norwegian group and our patients from Denmark, the difference is striking. It may, however, be explained by a variety of factors. First, baseline age and duration of diabetes was higher in our group. Second, in the Norwegian study only one retinal image was obtained for each eye and grading was not done at a grading centre. In the Norwegian cohort, the incidence of non-proliferative retinopathy equalled the $10 \%$ incidence of proliferative retinopathy. Given the limitations above, proliferative retinopathy may have been misclassified in
Table 2 Overall and sex-specific characteristics of patients at follow-up

Continuous data are presented as means with standard deviations; categorical data are presented as percentages

\begin{tabular}{lllll}
\hline Characteristic & Overall $(n=201)$ & Men $(n=120)$ & Women $(n=81)$ & $p$ value \\
\hline Age (years) & $58.9 \pm 10.1$ & $58.4 \pm 9.7$ & $59.6 \pm 10.7$ & 0.43 \\
Duration of diabetes (years) & $44 \pm 8$ & $44 \pm 7$ & $45 \pm 8$ & 0.17 \\
History of smoking (\%) & 59.7 & 68.6 & 46.3 & 0.002 \\
Neuropathy (\%) & 52.7 & 57.9 & 45.0 & 0.07 \\
Macrovascular disease (\%) & 21.9 & 24.8 & 17.5 & 0.22 \\
HbA $_{\text {1c }}$ \%) & $7.7 \pm 1.1$ & $7.7 \pm 1.1$ & $7.7 \pm 1.0$ & 0.83 \\
Nephropathy (\%) & & & & 0.82 \\
Normoalbuminuria & 66.8 & 65.8 & 68.4 & \\
Microalbuminuria & 22.6 & 22.5 & 22.8 & 8.9 \\
Macroalbuminuria & 10.6 & 11.7 & 55.7 & 0.14 \\
Blood-pressure-lowering treatment (\%) & 62.0 & 66.1 & $146 \pm 22$ & 0.10 \\
Systolic blood pressure (mmHg) & $149 \pm 21$ & $151 \pm 20$ & $73 \pm 10$ & 0.01 \\
Diastolic blood pressure (mmHg) & $76 \pm 10$ & $77 \pm 10$ & 51.9 & 0.29 \\
Lipid-lowering treatment (\%) & 47.2 & 44.2 & & \\
Lipids & & & $5.0 \pm 0.9$ & 0.01 \\
Total cholesterol (mmol/l) & $4.8 \pm 0.9$ & $4.7 \pm 0.8$ & $2.3 \pm 0.6$ & $<0.001$ \\
HDL-cholesterol (mmol/l) & $2.1 \pm 0.6$ & $1.9 \pm 0.6$ & $2.4 \pm 0.7$ & 0.98 \\
LDL-cholesterol (mmol/1) & $2.4 \pm 0.7$ & $2.4 \pm 0.7$ & $1.0 \pm 0.6$ & 0.07 \\
Triacylglycerol (mmol/1) & $1.1 \pm 0.6$ & $1.1 \pm 0.6$ & \\
\hline
\end{tabular}


Table 3 Overall and sex-specific level of retinopathy in the worst eye at follow-up

\begin{tabular}{|c|c|c|c|c|c|c|}
\hline \multirow[t]{2}{*}{ ETDRS level } & \multicolumn{2}{|c|}{ Overall $(n=201)$} & \multicolumn{2}{|c|}{ Men $(n=121)$} & \multicolumn{2}{|c|}{ Women $(n=80)$} \\
\hline & $\%$ & $n$ & $\%$ & $n$ & $\%$ & $n$ \\
\hline No retinopathy & 3.0 & 6 & 2.5 & 3 & 3.8 & 3 \\
\hline 10 & 3.0 & 6 & 2.5 & 3 & 3.8 & 3 \\
\hline Non-proliferative retinopathy & 45.8 & 92 & 44.7 & 54 & 47.8 & 38 \\
\hline 20 & 13.9 & 28 & 15.7 & 19 & 11.3 & 9 \\
\hline 31 & 12.4 & 25 & 9.9 & 12 & 16.3 & 13 \\
\hline 37 & 7.5 & 15 & 7.4 & 9 & 7.5 & 6 \\
\hline 43 & 10.5 & 21 & 9.9 & 12 & 11.3 & 9 \\
\hline 47 & 1.5 & 3 & 1.7 & 2 & 1.3 & 1 \\
\hline Proliferative retinopathy & 51.2 & 103 & 52.9 & 64 & 48.8 & 39 \\
\hline 60 & 8.0 & 16 & 9.1 & 11 & 6.3 & 5 \\
\hline 62 & 2.0 & 4 & 2.5 & 3 & 1.3 & 1 \\
\hline 63 & 8.5 & 17 & 8.3 & 10 & 8.8 & 7 \\
\hline 64 & 4.5 & 9 & 5.8 & 7 & 2.5 & 2 \\
\hline 65 & 19.4 & 39 & 18.2 & 22 & 21.3 & 17 \\
\hline 71 & 4.0 & 8 & 5.0 & 6 & 2.5 & 2 \\
\hline 85 & 5.0 & 10 & 4.1 & 5 & 6.3 & 5 \\
\hline
\end{tabular}

some patients and, subsequently, the incidence of proliferative retinopathy may have been underestimated.

Proliferative diabetic retinopathy is the leading cause of blindness in type 1 diabetes [3, 18], but it has also been associated with non-ocular complications such as heart attack, stroke, nephropathy, amputations and mortality [3, 19-21]. In an age- and sex-adjusted model of 16 year follow-up of type 1 diabetic WESDR patients, Klein and associates found an all-cause mortality hazard ratio of 5.53 (95\% CI 3.06-9.99) for patients with proliferative retinopathy at baseline [19]. In a recent publication we were able to expand this finding by demonstrating that mortality depended upon proliferative retinopathy as well as proteinuria [21]. The 25 year survival rate was significantly lower $(p<0.001)$ for patients who had both $(16.7 \%)$ as compared with patients with: neither $(66.4 \%)$; proliferative retinopathy only $(48.2 \%)$; or proteinuria only $(33.3 \%)$.

In our study, non-proliferative retinopathy was identified as a major risk factor for incident proliferative retinopathy. Patients with baseline non-proliferative retinopathy were five times more likely to develop proliferative retinopathy than patients without. This is not surprising given the natural history of proliferative retinopathy. In the WESDR, there was a significant trend of progression to proliferative retinopathy with increasing baseline level of retinopathy [8]. For patients with moderate non-proliferative retinopathy in both eyes (ETDRS level 41 ), the 4 year progression to proliferative retinopathy was $42.4 \%$.

We identified glycaemic regulation as an important predictor of proliferative retinopathy. The OR for incident

Table 4 Logistic regression indicating ORs for 25 year progression to proliferative retinopathy according to baseline risk factors in age- and sexadjusted and multivariate models

\begin{tabular}{llcc}
\hline Risk factor & Increment & Age- and sex-adjusted model OR (95\% CI) & Multivariate model OR (95\% CI) \\
\hline Duration & 10 years & $1.02(0.59-1.78)$ & $0.69(0.35-1.36)$ \\
$\mathrm{HbA}_{1}$ & $1 \%$ & $1.71(0.95-3.08)$ & $2.14(1.06-4.31)^{*}$ \\
Proteinuria & vs no proteinuria & $6.82(0.73-64.1)$ & $5.17(0.49-54.3)$ \\
History of smoking & vs never smoked & $1.34(0.71-2.53)$ & $1.37(0.64-2.91)$ \\
Systolic BP & $10 \mathrm{mmHg}$ & $1.06(0.89-1.26)$ & $0.91(0.69-1.20)$ \\
Diastolic BP & $10 \mathrm{mmHg}$ & $1.16(0.89-1.51)$ & $1.31(0.86-1.99)$ \\
BMI & $1 \mathrm{~kg} / \mathrm{m}^{2}$ & $1.09(0.95-1.24)$ & $1.01(0.86-1.20)$ \\
Non-proliferative retinopathy & vs no retinopathy & $4.83(2.21-10.5)^{*}$ & $4.61(1.94-11.0)^{*}$ \\
Maculopathy & vs no maculopathy & $5.71(0.62-52.3)$ & $5.61(0.54-58.5)$
\end{tabular}

$* p<0.05$ 
proliferative retinopathy at the 25 year follow-up examination was 2.14 (95\% CI 1.06-4.31) for each $1 \%$ increase in baseline $\mathrm{HbA}_{1}$. Thus, given similarities in other risk factors, the risk of proliferative retinopathy was 9.8 times higher for a patient who had a baseline $\mathrm{HbA}_{1}$ of $10.0 \%$ than for a patient with $\mathrm{HbA}_{1} 7.0 \%$. The effect of hyperglycaemia on incident proliferative retinopathy is well known from other long-term studies of type 1 diabetes [5, 17]. Furthermore, in the Diabetes Control and Complications Trial the benefits of strict glycaemic regulation were demonstrated in type 1 diabetes [22]. Progression to severe non-proliferative retinopathy or proliferative retinopathy was reduced by $47 \%$ among intensively treated patients $(p=0.01)$.

Only $3 \%$ of our patients had no signs of retinopathy at follow-up. This is in accordance with the very high rate of diabetic retinopathy found at the baseline examination in the WESDR study in 1984 [1]. Diabetic retinopathy was present in $97.5 \%$ of all patients who had had diabetes for longer than 15 years. The similarity of these results is interesting given that 23 years have passed. A lower prevalence of retinopathy in our study would have been expected.

Other possible risk factors for incident proliferative retinopathy were examined. In the multivariate analysis, proliferative retinopathy could not be predicted by duration of diabetes, proteinuria, smoking, blood pressure (systolic and diastolic), BMI or maculopathy. Results from other long-term follow-up studies are conflicting. Skrivarhaug et al. confirmed that proliferative retinopathy was independent of duration of diabetes, smoking and blood pressure [17]. In the 25 year follow-up of the WESDR cohort, however, proliferative retinopathy was predicted by baseline proteinuria, systolic blood pressure and BMI but remained unrelated to diastolic blood pressure and smoking [5]. In the EURODIAB Prospective Complications Study, risk factors for proliferative retinopathy were examined in a 7 year follow-up [23]. In EURODIAB, glycaemic regulation and baseline retinopathy were confirmed as risk factors for incident proliferative retinopathy, as was albumin excretion rate.

Fortunately, recent studies indicate that the incidence of proliferative retinopathy is decreasing in type 1 diabetes [15, $16,24]$. In a Danish study, the 20 year cumulative incidence of proliferative retinopathy was $31.2 \%$ in patients diagnosed in 1965-1969, compared with $12.5 \%$ in patients diagnosed in 1979-1984 [24]. The huge improvements in diabetes care with better control of blood glucose (i.e. with home monitoring and designer insulin formulations) and blood pressure are the most likely reasons for this improvement.

The major strengths of this study are the populationbased design and the long follow-up period. In many studies, incidence rates are overestimated because of referral bias. Studies which only include patients referred to specialist units may not be representative of the entire group of interest. Looking at complications in patients with a median duration of diabetes of 43 years makes it possible to evaluate the long-term impact of the disease more accurately.

Limitations must be considered. First, survival bias is a major concern. Patients who participated at the follow-up examination had all survived more than 30 years of diabetes. Many risk factors of interest have been associated with mortality [20], as has proliferative retinopathy [19, 21]. This may attenuate the association of proliferative retinopathy and risk factors such as blood pressure, proteinuria, smoking, BMI and maculopathy. Second, at the baseline examination the level of retinopathy was evaluated from ophthalmoscopy as fundus photographs were not available. If imaging had been on hand, the retinopathy grading would have been more accurate and adjustments for different levels of non-proliferative retinopathy would have been possible. Finally, our study is also limited by the lack of data on lipids and bloodpressure-lowering therapy at baseline as well as intermediary risk-factor and endpoint assessments during the long follow-up period.

To conclude, diabetic retinopathy is almost universal in long-surviving type 1 diabetic patients. Glycaemic regulation and non-proliferative retinopathy are important risk factors for proliferative retinopathy. Good glycaemic control is crucial and attending regular eye examinations may reduce the burden of visual impairment in type 1 diabetes.

Acknowledgements This work was supported by grants from the Velux Foundation, Danish Eye Health Society, Sehested Hansen's Foundation, Danish Diabetes Association, Synoptik Foundation, The A. P. Møller Foundation for the Advancement of Medical Science, The Danish Society of Ophthalmology and the Institute of Clinical Research at University of Southern Denmark.

Duality of interest The authors declare that there is no duality of interest associated with this manuscript.

\section{References}

1. Klein R, Klein BE, Moss SE, Davis MD, DeMets DL (1984) The Wisconsin epidemiologic study of diabetic retinopathy. II. Prevalence and risk of diabetic retinopathy when age at diagnosis is less than 30 years. Arch Ophthalmol 102:520-526

2. Sjolie AK, Stephenson J, Aldington S et al (1997) Retinopathy and vision loss in insulin-dependent diabetes in Europe. The EURODIAB IDDM Complications Study. Ophthalmology 104:252-260

3. Klein R, Klein BE (1995) Vision disorders in diabetes. In: Diabetes in America, 2nd edn. National Institutes of Health, Bethesda, MD, pp 1-36

4. Moss SE, Klein R, Klein BE (1994) Ten-year incidence of visual loss in a diabetic population. Ophthalmology 101:1061-1070 
5. Klein R, Knudtson MD, Lee KE, Gangnon R, Klein BE (2008) The Wisconsin Epidemiologic Study of Diabetic Retinopathy: XXII the twenty-five-year progression of retinopathy in persons with type 1 diabetes. Ophthalmology 115:1859-1868

6. Sjolie AK (1985) Ocular complications in insulin treated diabetes mellitus. An epidemiological study. Acta Ophthalmol Suppl 172:1-77

7. Green A, Hauge M, Holm NV, Rasch LL (1981) Epidemiological studies of diabetes mellitus in Denmark. II. A prevalence study based on insulin prescriptions. Diabetologia 20:468-470

8. Klein R, Klein BE, Moss SE, Davis MD, DeMets DL (1989) The Wisconsin Epidemiologic Study of Diabetic Retinopathy. IX. Four-year incidence and progression of diabetic retinopathy when age at diagnosis is less than 30 years. Arch Ophthalmol 107:237243

9. Klein R, Klein BE, Magli YL et al (1986) An alternative method of grading diabetic retinopathy. Ophthalmology 93:1183-1187

10. Anonymous (1991) Grading diabetic retinopathy from stereoscopic color fundus photographs - an extension of the modified Airlie House classification. ETDRS report number 10. Early Treatment Diabetic Retinopathy Study Research Group. Ophthalmology 98:786-806

11. Anonymous (1991) Fundus photographic risk factors for progression of diabetic retinopathy. ETDRS report number 12. Early Treatment Diabetic Retinopathy Study Research Group. Ophthalmology 98:823-833

12. Klein BE, Davis MD, Segal P et al (1984) Diabetic retinopathy. Assessment of severity and progression. Ophthalmology 91:10-17

13. Kolovou GD, Anagnostopoulou KK, Damaskos DS et al (2009) Gender differences in the lipid profile of dyslipidemic subjects. Eur J Intern Med 20:145-151

14. Krolewski AS, Warram JH, Rand LI, Christlieb AR, Busick EJ, Kahn CR (1986) Risk of proliferative diabetic retinopathy in juvenile-onset type I diabetes: a 40-yr follow-up study. Diabetes Care 9:443-452
15. Nordwall M, Bojestig M, Arnqvist HJ, Ludvigsson J (2004) Declining incidence of severe retinopathy and persisting decrease of nephropathy in an unselected population of Type 1 diabetes-the Linkoping Diabetes Complications Study. Diabetologia 47:1266-1272

16. Pambianco G, Costacou T, Ellis D, Becker DJ, Klein R, Orchard TJ (2006) The 30-year natural history of type 1 diabetes complications: the Pittsburgh Epidemiology of Diabetes Complications Study experience. Diabetes 55:1463-1469

17. Skrivarhaug T, Fosmark DS, Stene LC et al (2006) Low cumulative incidence of proliferative retinopathy in childhoodonset type 1 diabetes: a 24 -year follow-up study. Diabetologia 49:2281-2290

18. Anonymous (1987) Indications for photocoagulation treatment of diabetic retinopathy: Diabetic Retinopathy Study Report no. 14. The Diabetic Retinopathy Study Research Group. Int Ophthalmol Clin 27:239-253

19. Klein R, Klein BE, Moss SE, Cruickshanks KJ (1999) Association of ocular disease and mortality in a diabetic population. Arch Ophthalmol 117:1487-1495

20. Klein R, Moss SE, Klein BE, DeMets DL (1989) Relation of ocular and systemic factors to survival in diabetes. Arch Intern Med 149:266-272

21. Grauslund J, Green A, Sjolie AK (2008) Proliferative retinopathy and proteinuria predict mortality rate in type 1 diabetic patients from Fyn County, Denmark. Diabetologia 51:583-588

22. Anonymous (1993) The effect of intensive treatment of diabetes on the development and progression of long-term complications in insulin-dependent diabetes mellitus. The Diabetes Control and Complications Trial Research Group. N Engl J Med 329:977-986

23. Porta M, Sjoelie AK, Chaturvedi N et al (2001) Risk factors for progression to proliferative diabetic retinopathy in the EURODIAB Prospective Complications Study. Diabetologia 44:2203-2209

24. Hovind P, Tarnow L, Rossing K et al (2003) Decreasing incidence of severe diabetic microangiopathy in type 1 diabetes. Diabetes Care 26:1258-1264 